\title{
Finite element analysis of stimuli- responsive mesoscopic hydrogel via ultrafast laser processing (Withdrawal Notice)
}

\author{
Yufeng Tao, Xuhao Fan, Wei Xiong, Song Cai, Chunsan \\ Deng, et al.
}

Yufeng Tao, Xuhao Fan, Wei Xiong, Song Cai, Chunsan Deng, Jing Long, "Finite element analysis of stimuli-responsive mesoscopic hydrogel via ultrafast laser processing (Withdrawal Notice)," Proc. SPIE 11170, 14th National Conference on Laser Technology and Optoelectronics (LTO 2019), 111703R (17 May 2019); doi: 10.1117/12.2534000

SPIE Event: Fourteenth National Conference on Laser Technology and Optoelectronics, 2019, Shanghai, China 


\section{Finite element analysis of stimuli-responsive mesoscopic hydrogel via ultrafast laser processing (Withdrawal Notice)}

Yufeng Tao, Xuhao Fan, Wei Xiong, Song Cai, Chunsan Deng, Jing Long

Huazhong Univ. of Science and Technology (China)

Proceedings Volume 11170, 14th National Conference on Laser Technology and Optoelectronics (LTO 2019); $111703 R$ (2019) https://doi.org/10.1117/12.2534000

Event: Fourteenth National Conference on Laser Technology and Optoelectronics, 2019, Shanghai, China

Online Publication Date: 17 May 2019

Withdrawn from Publication: 24 July 2019

Publisher's Note: This paper, originally published on 17 May 2019, was withdrawn at the request of the authors. 